\title{
Facile Electrochemical Approach for the Production of Graphite Oxide with Tunable
}

Chemistry

Zhiming Tian ${ }^{1}$, Pei $Y^{1}$, Sean E. Lowe ${ }^{2}$, Anthony G. Pandolfo ${ }^{1}$, Thomas R. Gengenbach ${ }^{3}$, Kate M. Nairn ${ }^{1}$, Jingchao Song ${ }^{1}$, Xin Wang ${ }^{1}$, Yu Lin Zhong ${ }^{2 *}$, and Dan Li $^{1 *}$

Z. Tian, Y. Pei, Dr. A. G. Pandolfo, Dr. K. M. Nairn, J. Song, X. Wang and Prof. D. Li Department of Materials Science and Engineering

Monash University

VIC 3800, Australia

Email: dan.li2@monash.edu

S. E. Lowe, Dr. Y. L. Zhong,

Centre for Clean Environment and Energy

Gold Coast Campus, Griffith University, Gold Coast

QLD 4222, Australia

Email:y.zhong@griffith.edu.au

Dr. T. R. Gengenbach

CSIRO Manufacturing

Bayview Avenue, Clayton,

VIC 3168, Australia 


\begin{abstract}
Reproducible and in-depth studies of the electrochemical graphite intercalation and oxidation processes were carried out with the use of an electrochemical Tee-cell setup. The electrochemical method allowed simpler and greater controllability over the level of oxidation/functionalization, relative to the commonly employed chemical oxidation approach (e.g. the modified Hummers method). Extensive characterization was carried out to understand the properties of the electrochemically-derived graphite oxide (EGrO) and it was found that the abundance of each functionality is highly dependent on the electrochemical reaction time or by varying the concentration of the electrolyte (perchloric acid) employed. Notably, the amount of oxygen functional groups on EGrO could be as high as $30 \mathrm{wt}$ \%, but the degree of oxidation did not proceed beyond the generation of carbonyl species. The controllable oxidation level of the EGrO makes it an attractive precursor for many applications, such as electronics and nanocomposites.
\end{abstract}




\section{Introduction}

Chemically-derived graphite oxide (CGO) prepared via the oxidative chemical method (e.g. the Hummers method) is a popular precursor for production of graphene, because of its scalability, high yield and dispersibility in various solvents.[1-3] However, CGO not only suffers from contamination by metal ions (e.g. $\mathrm{K}^{+}$and $\mathrm{Mn}^{2+}$ ), [4, 5] but also the presence of inherent hole defects on the carbon basal plane,[6] caused by the strong oxidizing agents such as $\mathrm{KMnO}_{4}$, and $\mathrm{KClO}_{3},[7-9]$ which ultimately lead to a drastic drop in CGO's conductivity. Even after reduction by chemical or thermal method,[10-13] the electrical conductivity of the reduced CGO would be ultimately limited by those structural defects.[14, 15]

Alternatively, graphite oxide could be prepared via electrochemical methods.[16-18] Fundamentally, the intercalation of the graphite through the electrochemical oxidation process is similar to the chemical oxidation in which the graphite was first activated via the extraction of its electrons.[19] Although the electrochemical methods are considered to be greener compared to the chemical methods,[20, 21] a limitation of the paradigm to date has been the inability to tune the oxidation level of electrochemically-derived graphite oxide (EGrO) over a wide range. For instance, Bartosz et al. carried out electrochemical oxidation of graphite flakes held in a platinum mesh via linear sweep voltammetry, however, their EGrO displayed a relatively small degree of oxidation (C:O ratio of 9.8:1).[18] To this end, the structure and chemical tunability of EGrO are very critical for its eventual applications, as different oxidation degrees or oxygen functional groups will lead to considerable variation in its properties, including dispersibility, physical morphology, conductivity, toxicity and heavy ion adsorption behaviour.[22-25] 
A further limitation of previously reported electrochemical methods is the challenge to produce EGrO with high reproducibility. The difficulty lies in the ineffective distribution of electrical current to all graphite flakes for complete electrochemical intercalation and oxidation, especially after the initial graphite expansion caused by the intercalation and oxidation processes. Very frequently, the accompanied water/solvent electrolysis process exacerbates the detachment of multi-layered graphene flakes before the desired electrochemical reaction (e.g. oxidation) process can be achieved.[21, 26] To address these limitations, we describe a procedure for the controllable and reproducible synthesis of EGrO using a Tee-cell setup. In this study, the effects of charging time and electrolyte (perchloric acid) concentration were investigated, and the EGrO products were characterized in detail using X-ray diffraction (XRD), Nuclear magnetic resonance (NMR) spectroscopy, X-ray photoelectron spectroscopy (XPS), thermogravimetric analysis (TGA) and Raman spectroscopy.

\section{Experimental section}

Materials: The graphite flakes used in the experiments were purchased from SigmaAldrich (Product Number: 332461). The lateral size of the flakes was determined by scanning electron microscope (SEM) to be approximately $500 \mu$ m (Figure S1). Perchloric acid (11.6 M, 70 wt. \%) and sulfuric acid (18.0 M, 98 wt. \%) were obtained from Sigma-Aldrich and used as received. Other concentrations of acid were prepared by dilution with ultrapure Millipore water.

Sample Preparation: EGrO was prepared in a two-electrode Tee-cell purchased from Swagelok Eastern Australia (product No. PFA-820-3), as shown in Figure 1a. The anode was a graphite pellet (13 mm diameter and $185 \mu \mathrm{m}$ thickness) obtained by pressing $40 \mathrm{mg}$ of graphite flakes (under a pressure of 100 bar), without binder. Electrical connection to the 
graphite pellet was through a round platinum plate (13 mm diameter), backing the graphite pellet. Another round platinum plate was employed as the counter electrode and sandwiching a glass fiber filter membrane separator (Toyo Roshi Kaisha, Ltd. GA-55). A platinum wire was welded onto each platinum plate and connected to a VMP300 multichannel potentiostat/galvanostat (Princeton Applied Research) for all electrochemical reactions. During the EGrO preparation process, a constant current density, I = $50 \mu \mathrm{A} \mathrm{mg}^{-1}$, was applied to the cell for different times (from 0 to $170 \mathrm{ks}$ ). Various concentrations of perchloric acid (4.6 M, 7.0 M, 9.2 M, or 11.6 M) were employed as the electrolytes in the electrochemical process. After completion of the electrochemical oxidation process, the resulting solid was treated before further characterization. Four post-treatment processes were carried out: (a) characterized without any post-treatment, (b) immersed in water overnight, (c) copiously washed with water until the $\mathrm{pH}$ of EGrO dispersion was $~ 7$, and (d) washed samples dried under vacuum at $50{ }^{\circ} \mathrm{C}$ overnight. The samples mentioned in (b) and (c) were recovered via vacuum filtration using nitrocellulose membranes (pore size: $220 \mathrm{~nm}$, Merck Millipore) and characterized in their wetted form. For exfoliation of EGrO in water, $0.5 \mathrm{mg} / \mathrm{ml}$ aqueous dispersion of EGrO was prepared via ultrasonication (Branson Digital Sonifier 450D, 500 W, $30 \%$ amplitude) for 30 minutes and directly used for AFM characterization without centrifugation.

CGO was synthesized by a modified Hummers method as originally reported by Kovtyukhaova and colleagues.[27] In brief, graphite (5 g) was firstly placed in an $80{ }^{\circ} \mathrm{C}$ solution of concentrated sulfuric acid $(7.5 \mathrm{~mL}), \mathrm{P}_{2} \mathrm{O}_{5}(2.5 \mathrm{~g})$, and $\mathrm{K}_{2} \mathrm{~S}_{2} \mathrm{O}_{8}(2.5 \mathrm{~g})$ for preoxidation. The resultant dark blue mixture was kept at $80^{\circ} \mathrm{C}$ for $6 \mathrm{~h}$ and then cooled to room temperature, carefully diluted with ultrapure Millipore water, vacuum-filtered, and washed with water using glass fiber membrane until the filtrate became neutral. The pre-oxidized graphite was dried in an oven overnight at $50{ }^{\circ} \mathrm{C}$, and put in concentrated sulfuric acid (115 
$\left.\mathrm{mL}, 0{ }^{\circ} \mathrm{C}\right)$. Then $\mathrm{KMnO}_{4}(15 \mathrm{~g})$ was added gradually under continuous stirring for further oxidation and the temperature of the mixture was kept below $20^{\circ} \mathrm{C}$. The continuous stirring was maintained for $2 \mathrm{~h}$ after the mixture was heated to $35^{\circ} \mathrm{C} .230 \mathrm{~mL}$ water was added into the mixture; 15 mins later, $700 \mathrm{~mL}$ water and $12.5 \mathrm{~mL}$ hydrogen peroxide (30 wt.\%) were added to stop the oxidation reaction, and the mixture color became bright yellow. To remove metal ions, the mixture was washed with 1:10 $\mathrm{HCl}$ solution $(1.1 \mathrm{~L})$. Afterwards the mixture was centrifuged at 3000 rpm for 5 mins, and then the sediment was collected, redispersed in water and centrifuged. This process was repeated at least 3 times, until no sediment was found. The CGO dispersion was formed after the resulting solution was dialyzed for two weeks.

Characterization: The XRD patterns of EGrO and CGO films were recorded on a Philips 1130 X-ray diffractometer (40 kV, 25 mA, Cu Ka radiation, $\lambda=1.5418 \AA$ ) or Bruker $\mathrm{D}_{2}$ Phaser (30 kV, $10 \mathrm{~mA}, \mathrm{Cu} \mathrm{K \alpha}$ radiation, $\lambda=1.5418 \AA$ ) at room temperature. The data were collected from $5^{\circ}$ to $40^{\circ}$ at a scan rate of $2^{\circ} \mathrm{min}^{-1}$ and steps of $0.02^{\circ}$. The thermal properties of EGrO and CGO films were evaluated using a Perkin Elmer Thermogravimetric/Differential Thermal Analyzer (TG/DTA) 6300. The samples were heated under argon atmosphere from $30{ }^{\circ} \mathrm{C}$ to $700{ }^{\circ} \mathrm{C}$ at $5{ }^{\circ} \mathrm{C} \mathrm{min}^{-1}$. The electrical conductivity measurement for the EGrO films (35 mm diameter, $30 \mu \mathrm{m}$ thickness measured by micrometer) was carried out on a Jandel 4-point conductivity probe with a linear arrayed four-point head of $1 \mathrm{~mm}$ pin distance. SEM images of graphite flakes were obtained using a field-emission SEM (FEI Nova NanoSEM 450) with an operating voltage of $5 \mathrm{kV}$. XPS analysis for EGrO and CGO films was performed using an AXIS Ultra DLD spectrometer (Kratos Analytical Inc., Manchester, UK) with a monochromatic Al Ka source at a power of $180 \mathrm{~W}(15 \mathrm{kV} \times 12 \mathrm{~mA})$, a hemispherical analyser operating in the fixed analyser transmission mode and the standard aperture (analysis area: $0.3 \mathrm{~mm} \times 0.7 \mathrm{~mm}$ ). Survey 
spectra were acquired at a pass energy of $160 \mathrm{eV}$, high resolution spectra were recorded from individual peaks at $40 \mathrm{eV}$ pass energy (yielding a typical peak width for polymers of $1.0 \mathrm{eV}$ ). Each specimen was analysed at an emission angle of $0^{\circ}$ as measured from the surface normal. Assuming typical values for the electron attenuation length of relevant photoelectrons the XPS analysis depth (from which $95 \%$ of the detected signal originates) ranges between 5 and $10 \mathrm{~nm}$ for a flat surface. The accuracy associated with quantitative XPS is ca. $10 \%-15 \%$. Precision (ie. reproducibility) depends on the signal/noise ratio but is usually much better than $5 \%$. The latter is relevant when comparing similar samples. ${ }^{1} \mathrm{H}-{ }^{13} \mathrm{C}$ cross-polarization magic angle spinning (CP-MAS) solid state NMR spectra were collected at $100.6 \mathrm{MHz}$ on a Bruker Avance 400 (9.4 T magnet) with a $4 \mathrm{~mm}$ multinuclear solid state probe at room temperature (ca. $25^{\circ} \mathrm{C}$ ). Solid EGrO and CGO powders were packed into $\mathrm{ZrO}_{2}$ rotors with Kel-F caps. Conditions used: spin rate $10 \mathrm{kHz}$, SW 30 kHz (296 ppm); acquisition time 25 ms, 1500 data points, 3 sec recycle delay and 2 ms contact time. Spectra were collected and processed using Topspin 2.1. Raman spectra of EGrO and CGO were recorded in a confocal microscope system (WITec alpha 300R) with excitation by a $532 \mathrm{~nm}$ laser. The samples for Raman characterization were prepared by drop casting the aqueous dispersion of EGrO or CGO onto silicon wafers (with $285 \mathrm{~nm}$ of silicon oxide) which were cleaned by immersing in piranha solution (30 mins), washing with Millipore water and drying under nitrogen. AFM images of EGrO and exfoliated EGrO sheets were obtained by Bruker Dimension Icon system under tapping mode. The EGrO and exfoliated EGrO samples for AFM characterization were prepared by drop-casting EGrO and exfoliated EGrO aqeous dispersion ( $\sim 0.01 \mathrm{mg} / \mathrm{ml})$ on Si (100) wafer, previously cleaned in Piranha solution and nitrogen dried.

\section{Result and Discussions}

\subsection{Anodic oxidation of graphite}


The galvanostatic charging curves for various time periods are shown in Figure 1b. They exhibit a sequence of characteristic slopes and plateaus which can be divided into three charging segments. In general, the good overlap among the charging curves across the five different experiments suggests that the technique has good batch-to-batch reproducibility. This good reproducibility originated from the spatial confinement of graphite in the Tee-cell, thereby maintaining the electrical connectivity between graphite and Pt backing disc throughout the experiment.

The proposed overall reaction mechanism for the synthesis of EGrO is illustrated in Figure 1c. The initial segment (0 kilo-seconds (ks) to $6.5 \mathrm{ks})$ corresponds to the conversion of graphite into a graphite intercalation compound (GIC) with decreasing stage number. The final product for this segment is the acid salt $\mathrm{C}_{\mathrm{n}}\left(\mathrm{HClO}_{4}\right)_{\mathrm{x}}\left(\mathrm{ClO}_{4}\right)$ of a stage $1 \mathrm{HClO}_{4}$-GIC, where the maximum possible charge transfer to the conjugated $\pi$-system is achieved.[28] At this stage, all the charge was consumed for GIC formation, and practically no side reactions are involved as there was no sign of oxygen evolution (bubbling).[29] The reaction in this segment is summarized by Equation 1:

$$
\mathrm{nC}+(\mathrm{x}+1) \mathrm{HClO}_{4} \rightarrow \mathrm{C}_{\mathrm{n}}^{+} \mathrm{ClO}_{4}^{-} \cdot \mathrm{xHClO}_{4}+\mathrm{H}^{+}+\mathrm{e}^{-}
$$

The second stage (6.5 ks to $68 \mathrm{ks}$ ) corresponds to the conversion of stage $1 \mathrm{HClO}_{4}$-GIC complex into the oxidized form. Similarly, there was no sign of oxygen evolution (bubbling) in this segment. The sequential oxidation of the stage $1 \mathrm{HClO}_{4}-\mathrm{GIC}$ can be described by Equation 2 to 4.[30] In brief, the initial diol product may be further transformed to epoxy rings, and further oxidized via anodic C-C cleavage to form carbonyl groups. 

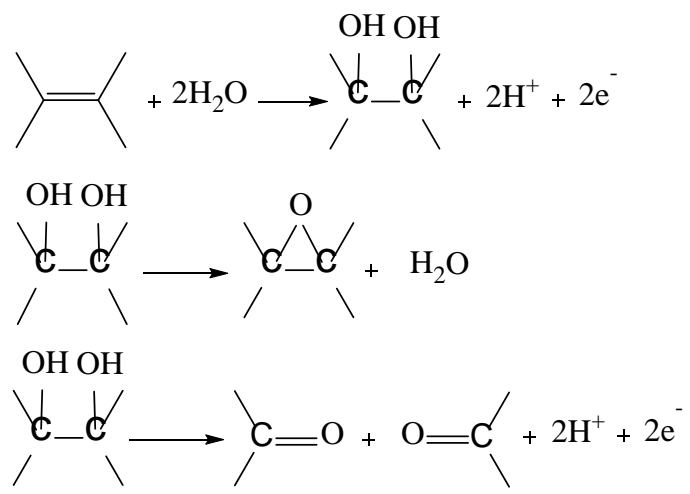

During the third charging segment (68 ks to $170 \mathrm{ks}$ ), oxygen evolution became dominant, due to the electrolysis of water at the EGrO or Pt plate surfaces, as described by Equation 5.[16, 31]

$$
\mathrm{H}_{2} \mathrm{O} \rightarrow 1 / 2 \mathrm{O}_{2}+2 \mathrm{H}^{+}+2 \mathrm{e}^{-}
$$

\subsection{Structural evolution of graphite to EGrO and the effect of post-treatment (washing) process}

XRD was used to monitor the structural evolution of graphite to $\mathrm{EGrO}$ and the effect of post-treatment (Figure 2). Figure 2a shows the XRD patterns of as-produced samples obtained directly from the electrolytic cell after various periods of anodic oxidation. After electrochemical charging for $6.5 \mathrm{ks}$, the as-produced EGrO sample showed a diffraction pattern typical for the stage $1 \mathrm{HClO}_{4}$-GIC complex. The (002) diffraction peak, at $23.2^{\circ}$, along with the (001) and (003) signals, at $11.8^{\circ}$ and $35.2^{\circ}$, respectively, can unambiguously be assigned to the stage $1 \mathrm{HClO}_{4}$-GIC complex, in good agreement with previous studies.[32] When the graphite was charged for $40 \mathrm{ks}$, the stage $1 \mathrm{HClO}_{4}$-GIC signals were broadened with reduction in their peak intensities, indicating the oxidative disordering of the GIC structure. After $60 \mathrm{ks}$, the (001) and (003) diffraction peaks disappear and the (002) diffraction peak becomes very broad and almost non-existent which indicates that the graphite lattice had become extensively disordered. 
After overnight immersion in water, the samples were characterized in their wetted form. A new diffraction peak, at around $8.5^{\circ}$, was observed for all the samples except for the 6.5 ks sample (Figure 2b). This diffraction peak can be attributed to the interlayer spacing of EGrO layers, containing oxygen-containing functional groups, which showed up in the XRD pattern after some of the intercalants were solvated. The intense (002) graphite peak observed for samples with less than $6.5 \mathrm{ks}$ of charging also indicated that the graphene layers were not oxidized during the initial GIC stage. After $60 \mathrm{ks}$ of charging, the (002) diffraction peak almost completely disappeared, indicating that almost no re-stacked multilayer graphene existed in these samples. In order to remove the residual acid completely, the immersed samples were repeatedly washed until $\mathrm{pH}$ of the dispersion is neutral and characterized in their wetted form as shown in Figure 2c. The washing process resulted in sharper and more intense EGrO peak at $8.5^{\circ}$. After drying overnight at $50{ }^{\circ} \mathrm{C}$, the EGrO peaks shifted to higher $2 \theta\left(12.3^{\circ}\right)$ due to the loss of water between the EGrO layers (Figure $2 \mathrm{~d}$ ). In comparison to the dried CGO sample with an interlayer spacing of $8.0 \AA$, the EGrO samples displayed smaller interlayer spacing of $6.5 \AA$ which is an indication of different oxygen-containing functional groups on EGrO and CGO.

The washed EGrO particles were briefly dispersed in water by stirring and dropcasted on Si wafer for AFM characterization. Figure S2 shows the AFM image of a typical EGrO flake of over $10 \mu \mathrm{m}$ in lateral size and over $160 \mathrm{~nm}$ thick. After ultrasonication in water for $30 \mathrm{~min}$, EGrO could be exfoliated to few-layered electrochemically-derived graphene oxide (EGO) with thickness down to $2 \mathrm{~nm}$ as shown in Figure S3a and S3b.

\subsection{Chemical properties and conductivity of EGrO}

All subsequent characterization was performed with EGrO samples that had been repeatedly washed and dried. Figure 3a displays the XPS survey scans of EGrO samples 
(after various oxidation periods) and CGO where the carbon and oxygen peaks are readily identified. The corresponding high-resolution C 1s spectra (Figure S4) were curve-fitted following the protocol described in Supporting Information in order to quantify different carbon/oxygen functional groups, specifically groups based on $\mathrm{C}-\mathrm{C}$ and $\mathrm{C}=\mathrm{C}$ bonds (no oxygen), $\mathrm{C}-\mathrm{O}, \mathrm{C}=\mathrm{O}$, and $\mathrm{O}-\mathrm{C}=\mathrm{O}$, respectively.[33] It can be seen from the $\mathrm{C}$ 1s scans (Figure 3b) and the tabulated data of the carbon components (Table 1) that the overall oxidation degree of EGrO samples (oxygen atomic ratio percentage) becomes higher with increasing charging time. This correlates well with the TGA results showing increasing weight loss (of functional groups) with increasing charging time (Figure 3c). For the EGrO samples after 60 ks, the oxidation degree of EGrO (C:O ratio of 3.0:1) approached that of CGO (C:O ratio of 2.5:1). Table 1 also shows that there was little change in the carboxyl content of EGrO over the course of anodic oxidation, indicating that the carboxyl functionalities (presumably at the edges of EGrO sheets) reached saturation at the very initial stage of the EGrO oxidation process $(6.5 \mathrm{ks})$. Interestingly, the concentration of carboxyls in highly oxidized EGrO (Figure S4a) was still significantly lower that of CGO (Figure S4b). Notably, the degree of basal plane oxidation on $\mathrm{EGrO}$, in the form of $\mathrm{C}=\mathrm{O}$ and $\mathrm{C}-\mathrm{O}$, reached the same level as that on CGO after $90 \mathrm{ks}$ of electrochemical oxidation. These results suggest that graphite oxidation occurred at the graphite edge sites before proceeding to the basal plane and, more importantly, the over-oxidation of $\mathrm{C}=\mathrm{O}$ and $\mathrm{C}-\mathrm{O}$ to $\mathrm{COOH}$ did not occur even after an extended period of electrochemical oxidation.

Since XPS is unable to accurately resolve the hydroxyl and epoxy contributions to the C-O peak, NMR was employed to probe the chemical differences between EGrO and CGO. As shown in the NMR data (Figure 3d), there are three major signals which can be assigned to epoxy, alcoholic, and graphitic carbons at around 61, 70, and $134 \mathrm{ppm}$, respectively, and two small peaks at 101 and 168 ppm, which are attributed to lactol and carboxyl groups.[34, 
35] In comparison, the relative intensity of the epoxy group, C-O-C, is greater for the EGrO than for the CGO. Besides, there is no observable carboxyl peak in the EGrO spectrum, while a small peak could be seen in the CGO spectrum; this is consistent with the XPS data in Figure 3b and Table 1.

Raman spectra were obtained on all EGrO samples, CGO and graphite, as shown in Figure 4a. The spectrum for graphite shows a sharp $G$ band at $1576 \mathrm{~cm}^{-1}$ due to first-order scattering of the $\mathrm{E}_{2 \mathrm{~g}}$ mode and a $2 \mathrm{D}$ band at $2722 \mathrm{~cm}^{-1}$ originated from second-order doubleresonant Raman scattering.[36, 37] EGrO and CGO samples exhibit a D band at $1355 \mathrm{~cm}^{-1}$, which is evidence for the presence of defects or functional groups on the graphene basal plane. [38] With increasing charging time, the intensity of the D band gradually increased and the $G$ band became broader, which suggests increasing number of functional groups were introduced to the EGrO samples as the reaction progressed.[39] The presence of 2D peaks in all EGrO samples (absent in CGO) and their lower D to G peak intensity ratios compared to those of CGO, implies fewer structural defects on the EGrO samples.

Figure 4b shows that EGrO samples had conductivities of a few $\mathrm{S} \mathrm{cm}^{-1}$, even after extensive electrochemical oxidation; these values are about seven orders of magnitude greater than that of CGO (typically $6 \times 10^{-7} \mathrm{~S} \mathrm{~cm}^{-1}$ ).[40] This is attributed to the fact that EGrO contained fewer hole defects (evident from Raman spectroscopy) and significantly fewer carboxyl groups (evident from XPS) which are typically found on edge/hole sites.

\subsection{Effect of perchloric acid concentration}

The charging curves for graphite in various concentrations of perchloric acid are shown in Figure 5a. For diluted perchloric acid (4.6 M), the graphite potential increased rapidly to about $2 \mathrm{~V}$, without going through the graphite oxidation plateau (at about $1.75 \mathrm{~V}$ ), 
and oxygen evolution was observed thereafter. For 7.0 M, 9.2 M and 11.6 M perchloric acid, the charging curves displayed three clear segments, indicating the sequential electrochemical processes of intercalation, oxidation and hydrolysis. At the end of the reaction (i.e. charged for $100 \mathrm{ks}$ ) and after repeated washing and complete drying, the structural differences for each EGrO sample were investigated using XRD (Figure 5b). In the $4.6 \mathrm{M}$ perchloric acid sample, a strong graphite peak and a weak graphite oxide peak were evident, indicating incomplete oxidation of the graphite. In contrast, EGrO samples prepared with 7.0 M, 9.2 M and 11.6 M perchloric acid displayed obvious graphite oxide peaks and negligible graphite peaks, indicating that the graphite was well oxidized in those sufficiently-concentrated solutions.

XPS was used to differentiate the samples' functionalities in more detail (Figure 5c, S5, S6, Table S1). It was observed that with the increase of perchloric acid concentration from 4.6 $\mathrm{M}$ to $11.6 \mathrm{M}$, the overall oxygen atomic percent (\%) increased from $19.1 \%$ to 25.0 \% (Figure 5c and S5), indicating that the oxidation level of EGrO is highly correlated to the concentration of perchlorate anions which determine their level of graphite intercalation.[30] Specifically, the percentage of C-O groups increased dramatically (25.7 \% to $37.3 \%$ ) when the concentration of perchloric acid was increased from 4.6 $\mathrm{M}$ to $9.2 \mathrm{M}$, but decreased (to $26.3 \%$ ) when the acid concentration was further increased to $11.6 \mathrm{M}$. This illustrates that the water content in the concentrated acid electrolyte (11.6 M) facilitated the graphite oxidation to diol groups according to Equation 2 to a certain extent (9.2 M). Interestingly, similar results have been observed in the water-enhanced chemical oxidation of graphite by Chen et al.[13d] In contrast, the carbonyl and carboxyl group ratio stayed relatively low (Figure 5c and S6) from $7.2 \mathrm{M}$ to $9.2 \mathrm{M}$. This implies that the electrochemical oxidation at these perchloric acid concentrations was limited to mild oxidation (to alcohol and epoxy groups) as described by Equation 2 and 3. In 11.6 M perchloric acid, the electrochemical oxidation of 
diol to two carbonyl groups was able to progress in accordance with Equation 4, and this is evident from the increased proportion of carbonyl group and decreased proportion of alcohol group. This clearly demonstrates that the simple selection of electrolyte concentration can control electrochemical oxidation degree and the eventual oxygen species on EGrO. Based on our study herein, the critical factor for increased level of graphite oxidation may be attributed to the electrochemical formation of stage-1 GIC and the sequential graphite oxidation under the appropriate electrolyte concentration and step-wise electrochemical reaction condition (i.e. galvanostatic).

\section{Conclusion}

In summary, EGrO can be readily produced in a reproducible and controlled manner in the Tee-cell setup for the detailed study of the electrochemical graphite intercalation and oxidation processes. Notably, the electrochemical oxidation process did not proceed to a higher degree of graphite oxidation (to produce carboxyl groups) even after extended period of reaction (in 11.6 M perchloric acid). Furthermore, we found that the degree of oxidation can be further limited by reducing the concentration of perchloric acid (9.2 $\mathrm{M}$ and below) in which case the oxidized groups were limited to alcohol and epoxy. In general, the structural defects and oxidation degree of EGrO were also found to be lower than in CGO. Therefore, the good structural integrity and controllable functional groups of the EGrO make it an attractive precursor for emerging graphene applications in electronics and nanocomposite materials.

\section{Supporting Information}

Supporting Information is available online or from the author.

\section{Acknowledgements}


The authors acknowledge funding support from Baosteel-Australia Joint Research and Development Centre (BA11006) and the Australian Research Council (DE 140101662). This work made use of the facilities at the Monash Centre for Electron Microscopy and Monash X-ray platform.

\section{References}

[1] Park S, Ruoff RS. Chemical methods for the production of graphenes. Nature Nanotech. 2009;4(4):217-24.

[2] Bai H, Li C, Shi G. Functional Composite Materials Based on Chemically Converted Graphene. Adv. Mater. 2011;23(9):1089-115.

[3] Sun Y, Wu Q, Shi G. Graphene based new energy materials. Energy Environ. Sci. 2011;4(4):1113-32.

[4] Kim F, Luo J, Cruz - Silva R, Cote LJ, Sohn K, Huang J. Self - Propagating Domino like Reactions in Oxidized Graphite. Adv. Funct. Mater. 2010;20(17):2867-73.

[5] Yeh C-N, Raidongia K, Shao J, Yang Q-H, Huang J. On the origin of the stability of graphene oxide membranes in water. Nature Chem. 2015;7(2):166-70.

[6] Erickson K, Erni R, Lee Z, Alem N, Gannett W, Zettl A. Determination of the Local Chemical Structure of Graphene Oxide and Reduced Graphene Oxide. Adv. Mater. 2010;22(40):4467-72.

[7] Hummers Jr WS, Offeman RE. Preparation of graphitic oxide. J. Am. Chem. Soc. 1958;80(6):1339.

[8] Brodie BC. On the atomic weight of graphite. Phil. Trans. R. Soc. Lond. 1859:249-59.

[9] Marcano DC, Kosynkin DV, Berlin JM, Sinitskii A, Sun Z, Slesarev A, et al. Improved Synthesis of Graphene Oxide. ACS Nano 2010;4(8):4806-14.

[10] Pei S, Cheng H-M. The reduction of graphene oxide. Carbon. 2012;50(9):3210-28.

[11] Lv W, Tang D-M, He Y-B, You C-H, Shi Z-Q, Chen X-C, et al. Low-Temperature Exfoliated Graphenes: Vacuum-Promoted Exfoliation and Electrochemical Energy Storage. ACS Nano 2009;3(11):3730-6.

[12] Zhang C, Lv W, Xie X, Tang D, Liu C, Yang Q-H. Towards low temperature thermal exfoliation of graphite oxide for graphene production. Carbon 2013;62:11-24.

[13] Chua CK, Pumera M. Chemical reduction of graphene oxide: a synthetic chemistry viewpoint. Chem. Soc. Rev. 2014;43(1):291-312.

[14] Hernandez Y, Nicolosi V, Lotya M, Blighe FM, Sun Z, De S, et al. High-yield production of graphene by liquid-phase exfoliation of graphite. Nature Nanotech. 2008;3(9):563-8.

[15] Tung VC, Allen MJ, Yang Y, Kaner RB. High-throughput solution processing of largescale graphene. Nature Nanotech. 2009;4(1):25-9.

[16] Besenhard J, Fritz H. Über die Reversibilität der elektrochemischen Graphitoxydation in Säuren. Z. Anorg. Allg. Chem. 1975;416(2):106-16.

[17] Boehm H, Eckel M, Scholz W. Untersuchungen am Graphitoxid V. Über den Bildungsmechanismus des Graphitoxids. Z. Anorg. Allg. Chem. 1967;353(5-6):236-42.

[18] Gurzęda B, Florczak P, Kempiński M, Peplińska B, Krawczyk P, Jurga S. Synthesis of graphite oxide by electrochemical oxidation in aqueous perchloric acid. Carbon 2016;100:540-5.

[19] Inagaki M, Iwashita N, Kouno E. Potential change with intercalation of sulfuric acid into graphite by chemical oxidation. Carbon 1990;28(1):49-55. 
[20] Low CTJ, Walsh FC, Chakrabarti MH, Hashim MA, Hussain MA. Electrochemical approaches to the production of graphene flakes and their potential applications. Carbon 2013;54:1-21.

[21] Yang S, Lohe MR, Müllen K, Feng X. New-Generation Graphene from Electrochemical Approaches: Production and Applications. Adv. Mater. 2016;28(29):6213-21.

[22] Moo JGS, Khezri B, Webster RD, Pumera M. Graphene Oxides Prepared by Hummers', Hofmann's, and Staudenmaier's Methods: Dramatic Influences on Heavy-Metal-Ion Adsorption. ChemPhysChem 2014;15(14):2922-9.

[23] Chng ELK, Pumera M. The Toxicity of Graphene Oxides: Dependence on the Oxidative Methods Used. Chem. Eur. J. 2013;19(25):8227-35.

[24] Dreyer DR, Park S, Bielawski CW, Ruoff RS. The chemistry of graphene oxide. Chem. Soc. Rev. 2010;39(1):228-40.

[25] Chen J, Zhang Y, Zhang M, Yao B, Li Y, Huang L, et al. Water-enhanced oxidation of graphite to graphene oxide with controlled species of oxygenated groups. Chem. Sci. 2016;7(3):1874-81.

[26] Zhong YL, Tian Z, Simon GP, Li D. Scalable production of graphene via wet chemistry: progress and challenges. Mater. Today 2014;18(2):73-8.

[27] Kovtyukhova NI, Ollivier PJ, Martin BR, Mallouk TE, Chizhik SA, Buzaneva EV, et al. Layer-by-layer assembly of ultrathin composite films from micron-sized graphite oxide sheets and polycations. Chem. Mater. 1999;11(3):771-8.

[28] Besenhard JO, Fritz HP. The electrochemistry of black carbons. Angew. Chem. Int. Ed. 1983;22(12):950-75.

[29] Fiang J, Beck F. Thermodynamic data for anodic solid state graphite oxidation products in 96\% sulphuric acid. Carbon 1992;30(2):223-8.

[30] Beck F, Jiang J, Krohn H. Potential oscillations during galvanostatic overoxidation of graphite in aqueous sulphuric acids. J. Electroanal. Chem. 1995;389(1-2):161-5.

[31] Jiang J. Thermodynamic data for anodic solid state graphite oxidation products in 71\%perchloric acid. Carbon 1993;31(5):663-6.

[32] Liu D, Lan G, Hu S, Wang H. X-ray diffraction and raman scattering studies of HClO 4graphite intercalation compounds. Carbon 1992;30(2):251-4.

[33] Beamson G, Briggs D. High resolution XPS of organic polymers: the Scienta ESCA300 database. Wiley, Chichester, 1992.

[34] Gao W, Alemany LB, Ci L, Ajayan PM. New insights into the structure and reduction of graphite oxide. Nature Chem. 2009;1(5):403-8.

[35] Su C, Acik M, Takai K, Lu J, Hao S-j, Zheng Y, et al. Probing the catalytic activity of porous graphene oxide and the origin of this behaviour. Nature Commun. 2012;3:1298.

[36] Ferrari AC, Meyer JC, Scardaci V, Casiraghi C, Lazzeri M, Mauri F, et al. Raman Spectrum of Graphene and Graphene Layers. Phys. Rev. Lett. 2006;97(18):187401.

[37] Dimiev AM, Bachilo SM, Saito R, Tour JM. Reversible Formation of Ammonium Persulfate/Sulfuric Acid Graphite Intercalation Compounds and Their Peculiar Raman Spectra. ACS Nano 2012;6(9):7842-9.

[38] Eckmann A, Felten A, Mishchenko A, Britnell L, Krupke R, Novoselov KS, et al. Probing the Nature of Defects in Graphene by Raman Spectroscopy. Nano Lett. 2012;12(8):3925-30.

[39] Yang D, Velamakanni A, Bozoklu G, Park S, Stoller M, Piner RD, et al. Chemical analysis of graphene oxide films after heat and chemical treatments by X-ray photoelectron and Micro-Raman spectroscopy. Carbon 2009;47(1):145-52.

[40] Xu Y, Bai H, Lu G, Li C, Shi G. Flexible Graphene Films via the Filtration of WaterSoluble Noncovalent Functionalized Graphene Sheets. J. Am. Chem. Soc. 2008;130(18):5856-7. 


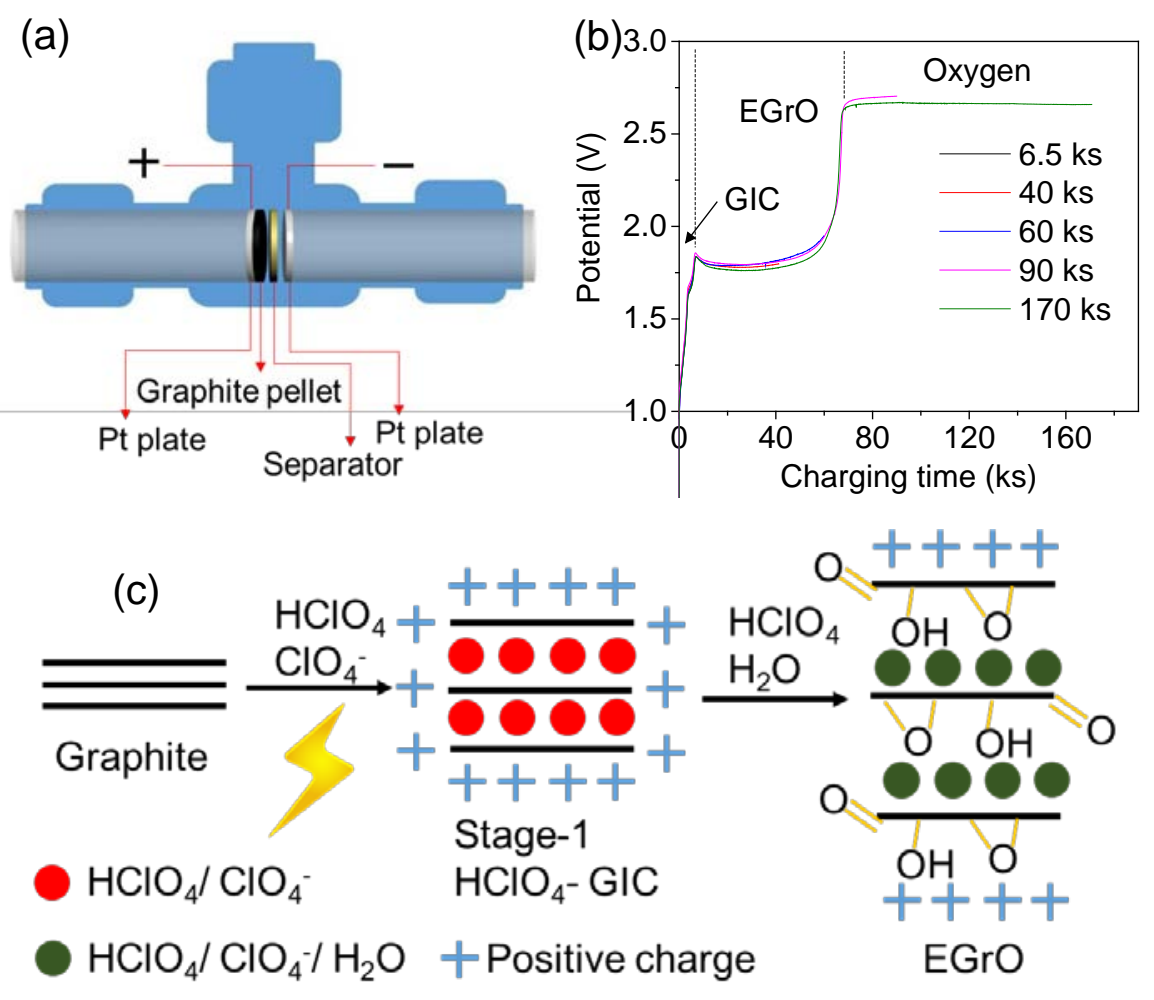

Figure 1. (a) Schematic drawing of the electrochemical Tee-cell setup and (b) potential recording during constant current oxidation of the graphite pellet in $11.6 \mathrm{M} \mathrm{HClO}_{4}$, where the curves for five different experiments of different durations (6.5 ks to $170 \mathrm{ks}$ ) are shown. Three different dominant reaction stages (GIC process, EGrO oxidation process, and oxygen evolution) are labeled. (c) Proposed mechanism of the formation of EGrO from graphite, showing the two distinct independent steps. The first step is the conversion of graphite into a stage- $1 \mathrm{HClO}_{4}$ graphite intercalation compound (GIC). The second step is the conversion of the stage- $1 \mathrm{HClO}_{4}$-GIC into EGrO. The solid black lines represent graphene layers. 
(a)

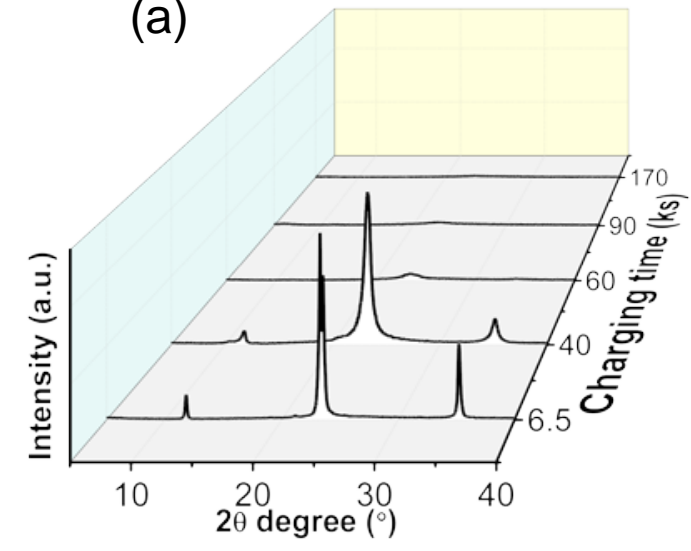

(c)

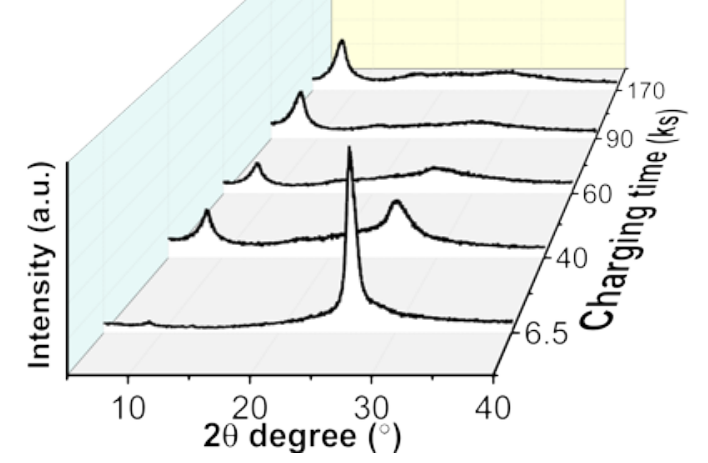

(b)

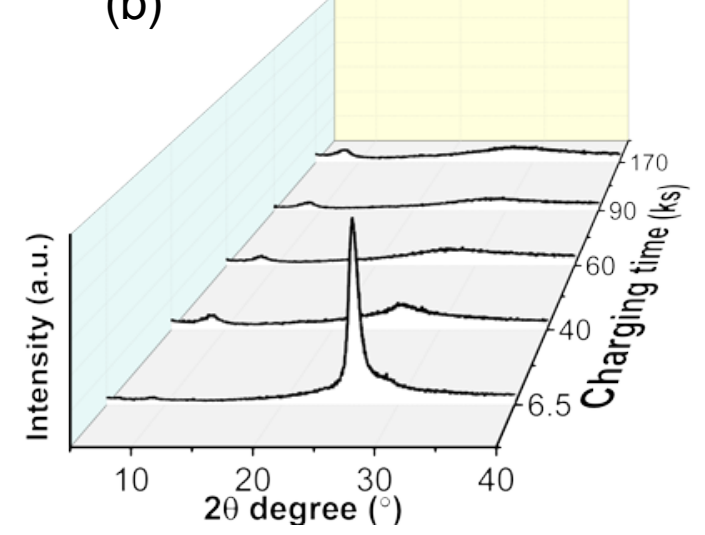

(d)

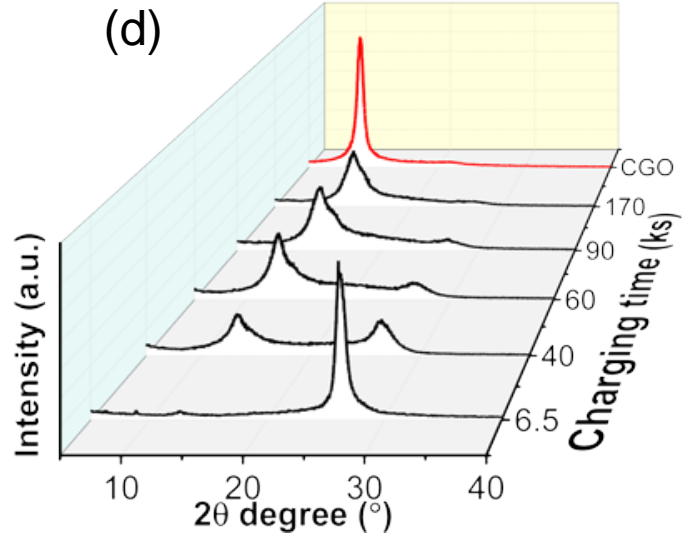

Figure 2. X-ray diffraction patterns of oxidized graphite after different charging periods and after various post-reaction treatments: (a) no post-reaction treatment, (b) after immersion in water overnight, (c) after repeated washing until neutral $\mathrm{pH}$ and (d) after repeated washing and overnight drying at $50{ }^{\circ} \mathrm{C}$. 

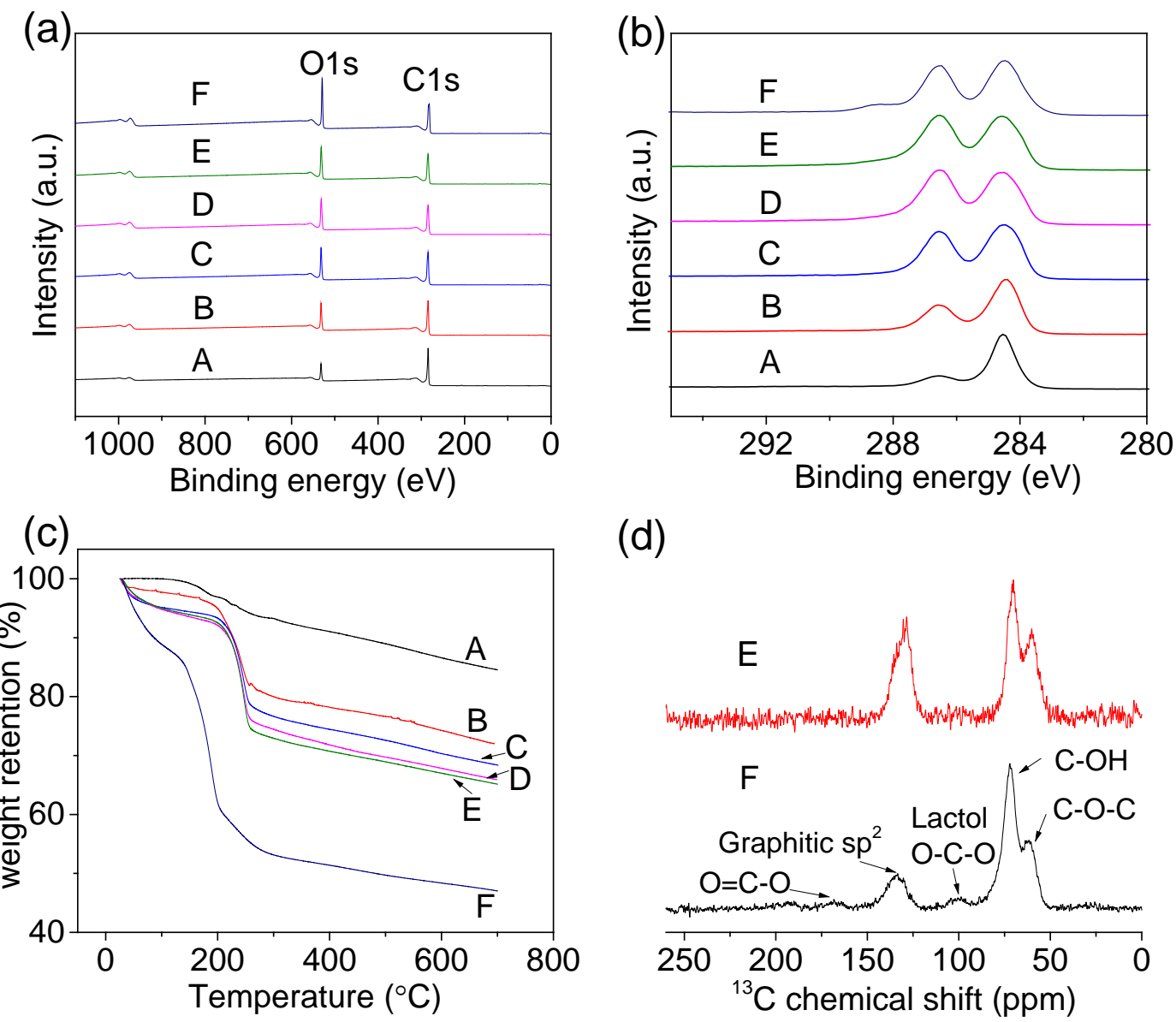

(d)

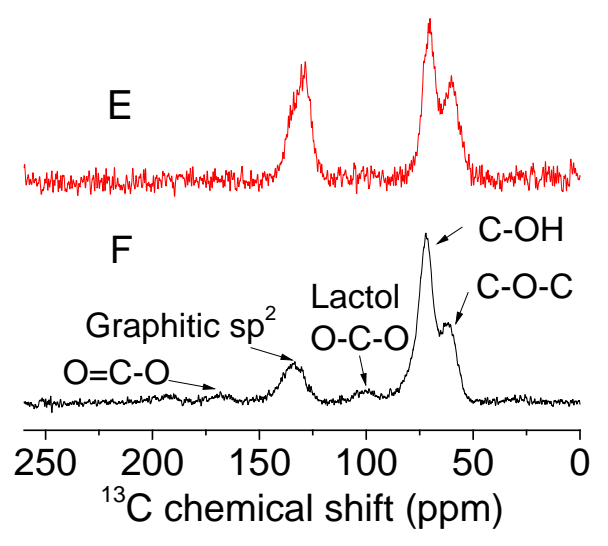

Figure 3. Comparison of chemical properties of EGrO as a function of charging time: $6.5 \mathrm{ks}$ (A), 40 ks (B), 60 ks (C), 90 ks (D), 170 ks (E) and CGO (F). (a) XPS survey scan spectra, (b) corresponding high-resolution $\mathrm{C}$ 1s spectra, (c) TGA plots, (d) ${ }^{1} \mathrm{H}-{ }^{13} \mathrm{C}$ CP-MAS NMR spectra of EGrO sample (charged for $170 \mathrm{ks}$ ) and CGO, with peak assignments from Gao et al.[34] 

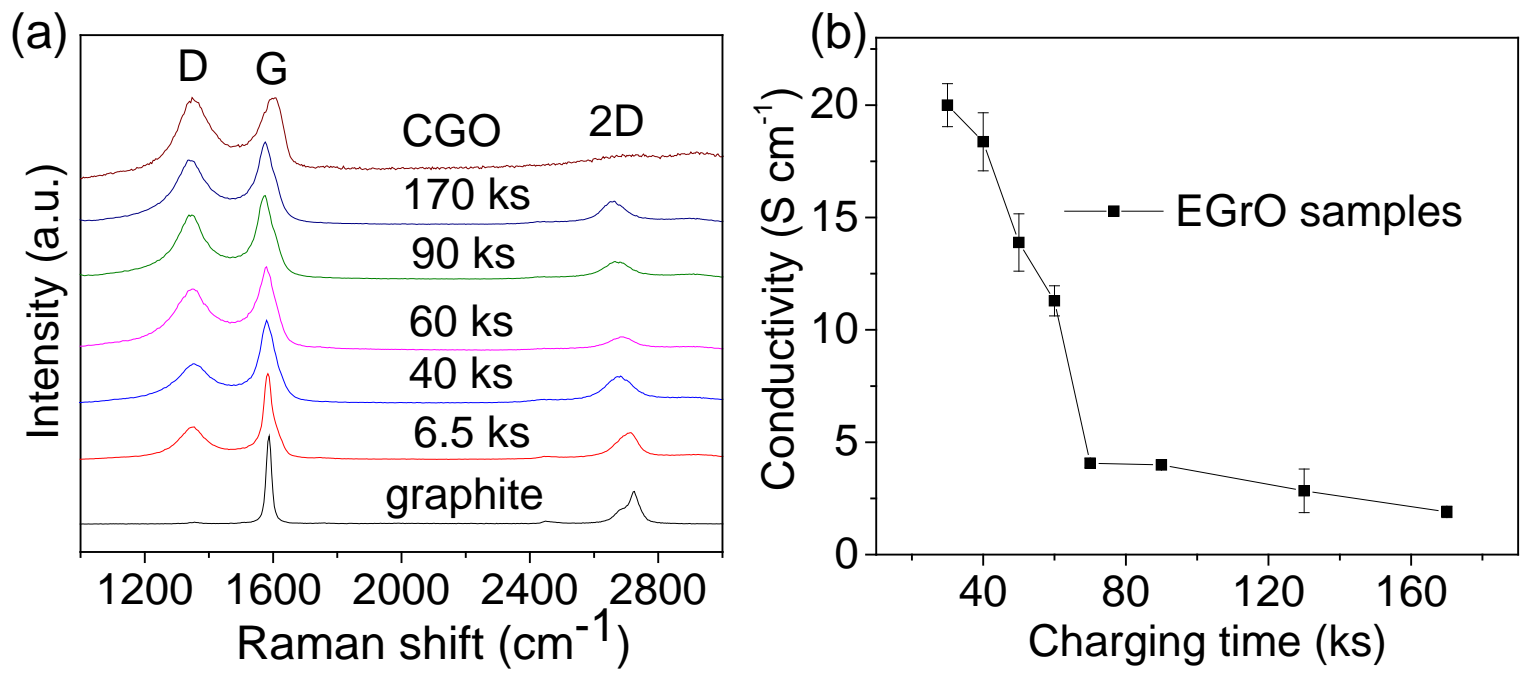

Figure 4. (a) Raman spectra of EGrO samples after specific charging times, graphite and CGO; (b) electrical conductivity of EGrO samples after specific charging times. 

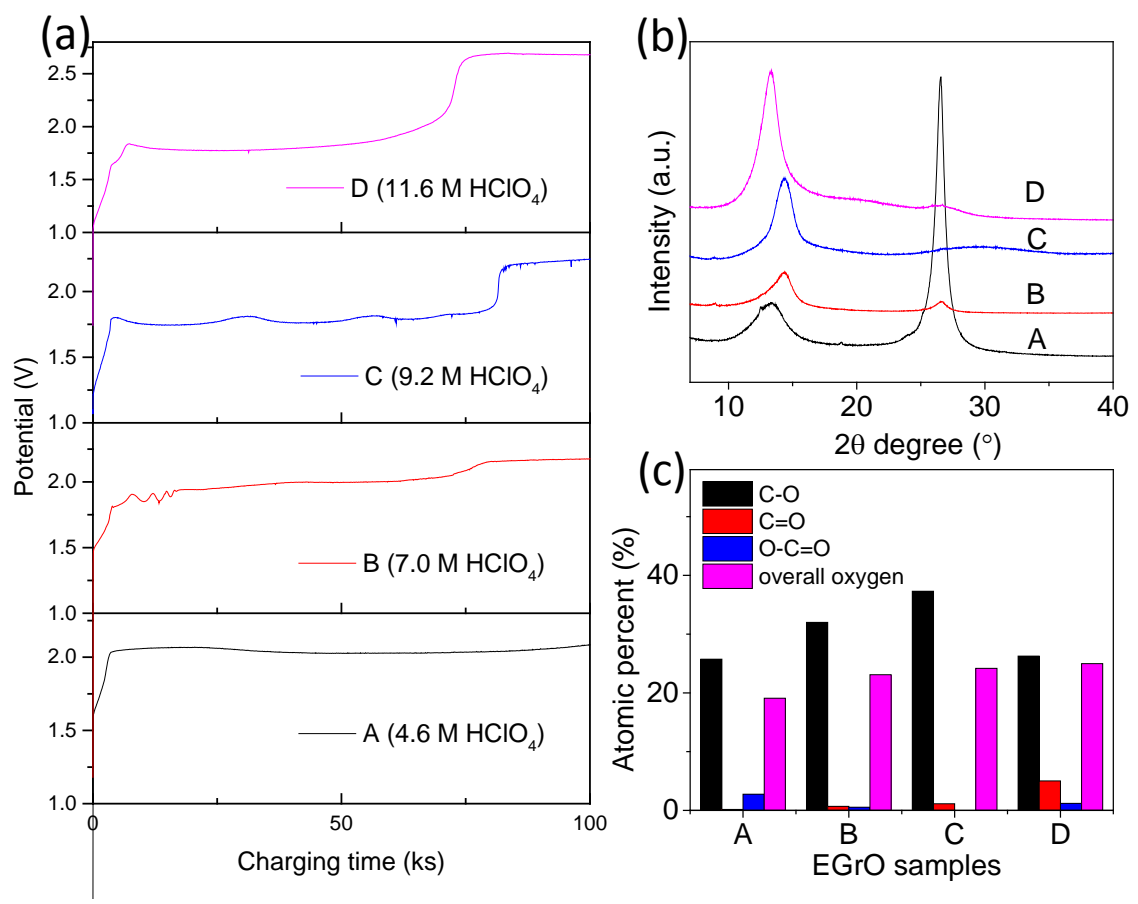

Figure 5. Synthesis and characterization of EGrO samples generated from various concentrations of perchloric acid: $4.6 \mathrm{M}$ (A), 7.0 M (B), $9.2 \mathrm{M}$ (C), and 11.6 M (D). (a) Potential vs charging time curves for different electrolyte conditions. (b) XRD patterns of samples after repeated washing and drying and (c) the atomic percentage of carbon components: alcohol, carbonyl, carboxyl and overall oxygen content from XPS spectrum curve fitting. 
Table 1. Percentage of XPS C 1s components, oxygen atomic content, C:O ratio and TGA plots for the EGrO samples and CGO

\begin{tabular}{|c|c|c|c|c|c|c|c|}
\hline \multirow{2}{*}{ Samples } & \multicolumn{4}{|c|}{ XPS C 1s components (\%) } & \multirow{2}{*}{$\begin{array}{c}\text { Oxygen atomic } \\
\text { percent (\%) }\end{array}$} & \multirow{2}{*}{ C 1s:0 1s } & \multirow{2}{*}{$\begin{array}{l}\text { TGA: weight loss } \\
(\%) \text { from } 100{ }^{\circ} \mathrm{C} \\
\text { to } 700^{\circ} \mathrm{C}\end{array}$} \\
\hline & $\mathrm{C}-\mathrm{C}$ & $\mathrm{O}-\mathrm{C}=\mathrm{O}$ & $\mathrm{C}=\mathrm{O}$ & $\mathrm{C}-\mathrm{O}$ & & & \\
\hline $\begin{array}{c}\text { EGrO, } 6.5 \\
\text { ks }\end{array}$ & 65.6 & 1.4 & 0.9 & 17.1 & 15.0 & 5.7 & 15.5 \\
\hline $\mathrm{EGrO}, 40 \mathrm{ks}$ & 54.4 & 1.0 & 2.8 & 21.8 & 20.1 & 4.0 & 25.7 \\
\hline EGrO, $60 \mathrm{ks}$ & 46.0 & 1.0 & 3.8 & 25.9 & 23.3 & 3.3 & 26.8 \\
\hline $\mathrm{EGrO}, 90 \mathrm{ks}$ & 42.5 & 1.3 & 4.9 & 26.4 & 24.9 & 3.0 & 28.9 \\
\hline $\begin{array}{l}\text { EGrO, } 170 \\
\text { ks }\end{array}$ & 42.3 & 1.3 & 5.1 & 26.3 & 25.1 & 3.0 & 29.9 \\
\hline CGO & 38.1 & 3.4 & 4.6 & 25.3 & 28.7 & 2.5 & 41.8 \\
\hline
\end{tabular}

Graphical abstract

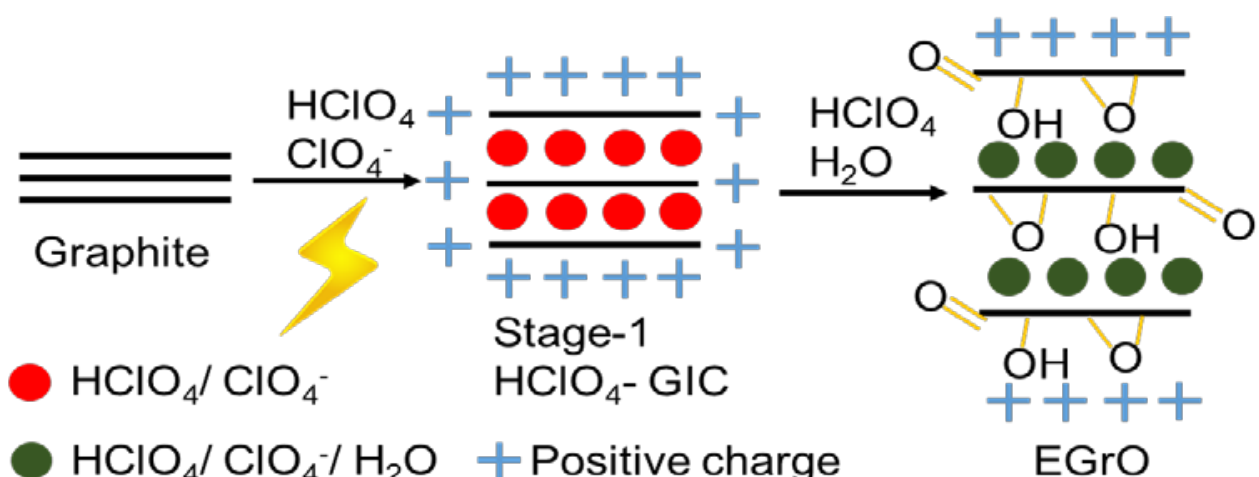

